\title{
Place-labelled Petri net controlled grammars
}

\author{
Nurhidaya Mohamad Jan ${ }^{\mathrm{a}}$, Sherzod Turaev ${ }^{\mathrm{b}, *}$, Wan Heng Fong ${ }^{\mathrm{c}}$, Nor Haniza Sarmin ${ }^{\mathrm{a}}$ \\ a Department of Mathematical Sciences, Faculty of Science, Universiti Teknologi Malaysia, \\ 81310 UTM Johor Bahru, Johor, Malaysia \\ b Department of Computer Science, Kulliyyah of Information and Communication Technology, \\ International Islamic University Malaysia, 53100 Kuala Lumpur, Malaysia \\ c Ibnu Sina Institute for Fundamental Science Studies, Universiti Teknologi Malaysia, \\ 81310 UTM Johor Bahru, Johor, Malaysia
}

*Corresponding author, e-mail: sherzod@iium.edu.my

Received 1 Dec 2014

Accepted 21 May 2017

\begin{abstract}
A place-labelled Petri net (pPN) controlled grammar is a context-free grammar equipped with a Petri net and a function which maps places of the net to the productions of the grammar. The language consists of all terminal strings that can be obtained by simultaneously applying the rules of multisets which are the images of the sets of the input places of transitions in a successful occurrence sequence of the Petri net. In this paper, we study the generative power and structural properties of pPN-controlled grammars. We show that pPN-controlled grammars have the same generative power as matrix grammars. Moreover, we prove that for each pPN-controlled grammar, we can construct an equivalent place-labelled ordinary net controlled grammar.
\end{abstract}

KEYWORDS: context-free grammars, computational power, structural properties

\section{INTRODUCTION}

Petri nets ${ }^{1}$, 'dynamic' bipartite directed graphs with two sets of nodes called places and transitions, provide an elegant and powerful mathematical formalism for modelling concurrent systems and their behaviour. Since Petri nets successfully describe and analyse the flow of information and the control of action in such systems, they can be very suitable tools for studying the properties of formal languages. If Petri nets are initially used as language generating/accepting tools ${ }^{2-8}$, in recent studies, they have been widely applied as regulation mechanisms for grammar systems ${ }^{9}$, automata ${ }^{10-15}$, and grammars ${ }^{16-30}$.

A Petri net controlled grammar is, in general, a context-free grammar equipped with a (place/ transition) Petri net and a function which maps transitions of the net to productions of the grammar. Then the language consists of all terminal strings that can be obtained by applying the sequence of productions which is the image of an occurrence sequence of the Petri net under the function. Several variants of Petri net controlled grammars have been introduced and investigated:

Refs. 18, 23 introduce $k$-Petri net controlled grammars and study their properties including generative power, closure properties, infinite hierarchies, etc.

Refs. 19, 21 consider a generalization of regularly controlled grammars: instead of a finite automaton, a Petri net is associated with a context-free grammar and it is required that the sequence of applied rules corresponds to an occurrence sequence of the Petri net, i.e., to sequences of transitions which can be fired in succession.

Refs. 20, 22 investigate grammars controlled by the structural subclasses of Petri nets, namely, state machines, marked graphs, causal nets, free-choice nets, asymmetric choice nets and ordinary nets. It is proven that the family of languages generated by (arbitrary) Petri net controlled grammars coincide with the family of languages generated by grammars controlled by free-choice nets.

Refs. 24, 25 continue the research on Petri net controlled grammars by restricting to (context-free, extended, or arbitrary) Petri nets with place capacities. A Petri net with place capacity regulates the defining grammar by permitting only those derivations where the number of each non-terminal in each sentential form is bounded by its capacity. It is shown that several families of languages generated 
by grammars controlled by extended Petri nets with place capacities coincide with the family of matrix languages of finite index.

In all above-mentioned variants of Petri net controlled grammars, the production rules of a core grammar are associated only with transitions of a control Petri net. Thus it is also interesting to consider the place labelling strategies with Petri net controlled grammars. Theoretically, it would complete the node labelling cases, i.e., we study the cases where the production rules are associated with places of a Petri net, not only with its transitions. Moreover, the place labelling makes it possible to consider parallel application of production rules in Petri net controlled grammars, which allows to develop formal language based models for synchronized/parallel discrete event systems.

Informally, a place-labelled Petri net controlled grammar (pPN-controlled grammar) is a contextfree grammar with a Petri net and a function which maps places of the net to productions of the grammar. The language consists of all terminal strings that can be obtained by parallelly applying of the rules of multisets which are the images of the sets of the input places of transitions in a successful occurrence sequence of the Petri net. In this paper, we study the effect of the place labelling strategies to the computational power, establish the lower and upper bounds for the families of languages generated by pPN-controlled grammars, and investigate their structural properties.

\section{PRELIMINARIES}

We assume that the reader is familiar with the basic concepts of formal language theory and Petri nets. In this section we only recall some notions, notation and results directly related to the current work. For more details see Refs. 2, 3, 31, 32 .

\section{Grammars}

Let $\Sigma$ be an alphabet. A string over $\Sigma$ is a sequence of symbols from the alphabet. The empty string is denoted by $\lambda$ which has no symbols. The set of all strings over the alphabet $\Sigma$ is denoted by $\Sigma^{*}$. A subset $L$ of $\Sigma^{*}$ is called a language. If $w=$ $w_{1} w_{2} w_{3}$ for some $w_{1}, w_{2}, w_{3} \in \Sigma^{*}$, then $w_{2}$ is called a substring of $w$. The length of a string $w$ is denoted by $|w|$, and the number of occurrences of a symbol $a$ in a string $w$ by $|w|_{a}$.

A multiset over an alphabet $\Sigma$ is a mapping $\pi$ : $\Sigma \rightarrow \mathbb{N}$. The alphabet $\Sigma$ is called the basic set of a multiset $\pi$ and the elements of $\Sigma$ is called the basic elements of a multiset $\pi$. A multiset $\pi$ over $\Sigma=$ $\left\{a_{1}, a_{2}, \ldots a_{n}\right\}$ is denoted by

$$
\pi=[\underbrace{a_{1}, \ldots, a_{1}}_{\pi\left(a_{1}\right)}, \underbrace{a_{2}, \ldots, a_{2}}_{\pi\left(a_{2}\right)}, \ldots, \underbrace{a_{n}, \ldots, a_{n}}_{\pi\left(a_{n}\right)}] .
$$

We also 'abuse' the set-membership notation by using it for multisets to write, for example, $a \in$ $[a, a, a, b]$ and $c \notin[a, a, a, b]$. The set of all multisets over $\Sigma$ is denoted by $\Sigma^{\oplus}$.

A context-free grammar is a quadruple $G=$ $(V, \Sigma, S, R)$, where $V$ and $\Sigma$ are disjoint finite sets of non-terminal and terminal symbols, respectively, $S \in V$ is the start symbol and a finite set $R \subseteq V \times$ $(V \cup \Sigma)^{*}$ is a set of (production) rules. Usually, a rule $(A, x)$ is written as $A \rightarrow x$. A rule of the form $A \rightarrow \lambda$ is called an erasing rule. A string $x \in(V \cup \Sigma)^{*}$ directly derives a string $y \in(V \cup \Sigma)^{*}$, written as $x \Rightarrow y$, if and only if there is a rule $r=(A, \alpha) \in R$ such that $x=$ $x_{1} A x_{2}$ and $y=x_{1} \alpha x_{2}$. The reflexive and transitive closure of $\Rightarrow$ is denoted by $\Rightarrow^{*}$. A derivation using the sequence of rules $\pi=r_{1} r_{2} \cdots r_{n}$ is denoted by $\stackrel{\pi}{\Longrightarrow}$ or $\stackrel{r_{1} r_{2} \cdots r_{n}}{\Longrightarrow}$. The language generated by $G$ is defined by $L(G)=\left\{w \in \Sigma^{*} \mid S \Rightarrow^{*} w\right\}$.

A matrix grammar is a quadruple $G=(V, \Sigma, S, M)$, where $V, \Sigma, S$ are defined as for a context-free grammar and $M$ is a finite set of matrices, which are finite strings over a set of context-free rules (or finite sequences of context-free rules). The language generated by $G$ is $L(G)=\left\{w \in \Sigma^{*} \mid S \stackrel{\pi}{\Rightarrow} w, \pi \in M^{*}\right\}$. The families of languages generated by matrix grammars without erasing rules and by matrix grammars with erasing rules are denoted by MAT and MAT $^{\lambda}$, respectively.

\section{Theorem 1 (Ref. 33)}

$$
\mathrm{CF} \subset \mathrm{MAT} \subset \mathrm{CS}, \mathrm{MAT} \subseteq \mathrm{MAT}^{\lambda} \subset \mathrm{RE},
$$

where $\mathbf{C F}$, CS and $\mathbf{R E}$ denote the families of contextfree, context-sensitive and recursively enumerable languages, respectively.

\section{Petri nets}

A Petri net (PN) is a construct $N=(P, T, F, \phi)$ where $P$ and $T$ are disjoint finite sets of places and transitions, respectively, $F \subseteq(P \times T) \cup(T \times P)$ is the set of directed arcs, $\phi: F \rightarrow \mathbb{N}$ is a weight function.

A Petri net can be represented by a bipartite directed graph with the node set $P \cup T$ where places are drawn as circles, transitions as boxes and arcs as arrows. The arrow representing an $\operatorname{arc}(x, y) \in F$ is labelled with $\phi(x, y)$; if $\phi(x, y)=1$, then the label is omitted. 
An ordinary net (ON) is a Petri net $N=$ $(P, T, F, \phi)$ where $\phi(x, y)=1$ for all $(x, y) \in F$. We omit $\phi$ from the definition of an ordinary net, written as $N=(P, T, F)$.

A mapping $\mu: P \rightarrow \mathbb{N}_{0}$ is called a marking. For each place $p \in P, \mu(p)$ gives the number of tokens in $p$. Graphically, tokens are drawn as small solid dots inside circles. The sets ${ }^{\circ} x=\{y \mid(y, x) \in F\}$ and $x^{\circ}=\{y \mid(x, y) \in F\}$ are called pre- and postsets of $x \in P \cup T$, respectively. For $X \subseteq P \cup T$, define ${ }^{\circ} X=\bigcup_{x \in X}{ }^{\circ} x$ and $X^{\circ}=\bigcup_{x \in X} x^{\circ}$. For $t \in T$ and $p \in P$, the elements of ${ }^{\circ} t^{\circ} p$ are called input places (transitions) and the elements of $t^{\circ} p^{\circ}$ are called output places (transitions) of $t p$.

A sequence of places and transitions $\rho=$ $x_{1} x_{2} \cdots x_{n}$ is called a path if and only if no place or transition except $x_{1}$ and $x_{n}$ appears more than once, and $x_{i+1} \in x_{i}^{\circ}$ for all $1 \leqslant i \leqslant n-1$.

A transition $t \in T$ is enabled by marking $\mu$ if and only if $\mu(p) \geqslant \phi(p, t)$ for all $p \in^{\circ} t$. In this case $t$ can occur(fire). Its occurrence transforms the marking $\mu$ into the marking $\mu^{\prime}$ defined for each place $p \in P$ by $\mu^{\prime}(p)=\mu(p)-\phi(p, t)+\phi(t, p)$. We write $\mu \rightarrow \mu^{\prime}$ to indicate that the firing of $t$ in $\mu$ leads to $\mu^{\prime}$. A marking $\mu$ is called terminal if in which no transition is enabled. A finite sequence $t_{1} t_{2} \cdots t_{k} \in T^{*}$, is called an occurrence sequence enabled at a marking $\mu$ and finished at a marking $\mu^{\prime}$ if there are markings $\mu_{1}, \mu_{2}, \ldots, \mu_{k-1}$ such that

$$
\mu \stackrel{t_{1}}{\rightarrow} \mu_{1} \stackrel{t_{2}}{\rightarrow} \ldots \stackrel{t_{k-1}}{\rightarrow} \mu_{k-1} \stackrel{t_{k}}{\rightarrow} \mu^{\prime} .
$$

In short this sequence can be written as $\mu \stackrel{t_{1} t_{2} \cdots t_{k}}{\longrightarrow} \mu^{\prime}$ or $\mu \stackrel{\nu}{\rightarrow} \mu^{\prime}$, where $v=t_{1} t_{2} \cdots t_{k}$. For each $1 \leqslant i \leqslant$ $k$, marking $\mu_{i}$ is called reachable from marking $\mu$. $\mathscr{R}(N, \mu)$ denotes the set of all reachable markings from a marking $\mu$.

A marked Petri net is a system $N=(P, T, F, \phi, \iota)$ where $(P, T, F, \phi)$ is a Petri net, $\iota$ is the initial marking.

A Petri net with final markings is a construct $N=(P, T, F, \phi, \iota, M)$ where $(P, T, F, \phi, \iota)$ is a marked Petri net and $M \subseteq \mathscr{R}(N, \iota)$ is a set of markings which are called final markings. An occurrence sequence $v$ of transitions is called successful for $M$ if it is enabled at the initial marking $\iota$ and finished at a final marking $\tau$ of $M$. If $M$ is understood from the context, we say that $v$ is a successful occurrence sequence.

\section{DEFINITIONS AND EXAMPLES}

In this section, we define a place-labelled Petri net controlled grammar, a derivation step, a successful derivation and the language of a place-labelled Petri net controlled grammar.

Definition 1 A place-labelled Petri net controlled grammar (pPN-controlled grammar) is a 7-tuple $G=(V, \Sigma, R, S, N, \beta, M)$ where $(V, \Sigma, R, S)$ is a context-free grammar, $N=(P, T, F, \phi, \iota)$ is a (marked) Petri net, $\beta: P \rightarrow R \cup\{\lambda\}$ is a placelabelling function and $M$ is a set of final markings.

Let $A \subseteq P$. We use the notation $\beta(A)$ and $\beta_{-\lambda}(A)$ to denote the multisets $[\beta(p) \mid p \in A]$ and $[\beta(p) \mid$ $p \in A, \beta(p) \neq \lambda]$, respectively.

Definition $2 x \in(V \cup \Sigma)^{*}$ directly derives $y \in(V \cup$ $\Sigma)^{*}$ with a multiset $\pi=\left[A_{i_{1}} \rightarrow \alpha_{i_{1}}, \ldots, A_{i_{k}} \rightarrow \alpha_{i_{k}}\right] \subseteq$ $R^{\oplus}$, written as $x \stackrel{\pi}{\Longrightarrow} y$, if and only if

$$
\begin{aligned}
& x=x_{1} A_{i_{1}} x_{2} A_{i_{2}} \cdots x_{k} A_{i_{k}} x_{k+1}, \\
& y=x_{1} \alpha_{i_{1}} x_{2} \alpha_{i_{2}} \cdots x_{k} \alpha_{i_{k}} x_{k+1},
\end{aligned}
$$

where $x_{j} \in(V \cup \Sigma)^{*}, 1 \leqslant j \leqslant k+1$, and $\pi=\beta_{-\lambda}\left({ }^{\circ} t\right)$ for some $t \in T$ enabled at a marking $\mu \in \mathscr{R}(N, \iota)$.

Definition 3 A derivation

$$
S \stackrel{\pi_{1}}{\Longrightarrow} w_{1} \stackrel{\pi_{2}}{\Longrightarrow} w_{2} \stackrel{\pi_{3}}{\Longrightarrow} \cdots \stackrel{\pi_{n}}{\Longrightarrow} w_{n}=w \in \Sigma^{*},
$$

where $\pi_{i} \subseteq R^{\oplus}, 1 \leqslant i \leqslant n$, is called successful if and only if $\pi_{i}=\beta_{-\lambda}\left({ }^{\circ} t_{i}\right)$ for some $t_{i} \in T, 1 \leqslant i \leqslant n$, and $t_{1} t_{2} \cdots t_{n} \in T^{*}$ is a successful occurrence sequence in $N$. For short, (1) can be written as $S \stackrel{\pi_{1} \pi_{2} \cdots \pi_{n}}{\Longrightarrow} w$.

Definition 4 The language generated by pPNcontrolled grammar $G$ consists of all strings $w \in \Sigma^{*}$ such that there is a successful derivation $S \stackrel{\pi_{1} \pi_{2} \cdots \pi_{n}}{\Longrightarrow}$ $w$ in $G$.

With respect to different labelling strategies and the definition of final marking sets, we can define various variants of place-labelled Petri net controlled grammars. In this work, we define the following variants.

Definition 5 A pPN-controlled grammar $G=(V, \Sigma$, $S, R, N, \beta, M)$ is called free, denoted by $f$, if a different label is associated with each place, and no place is labelled with the empty string; $\lambda$-free, denoted by $-\lambda$, if no place is labelled with the empty string; or arbitrary, denoted by $\lambda$, if no restriction is posed on the labelling function $\beta$.

Definition 6 A pPN-controlled grammar $G=(V, \Sigma$, $S, R, N, \beta, M)$ is called $r$-type if $M$ is the set of all reachable markings from the initial marking $i, M=$ $\mathscr{R}(N, \iota)$, or called $t$-type if $M \subseteq \mathscr{R}(N, \iota)$ is a finite set. 


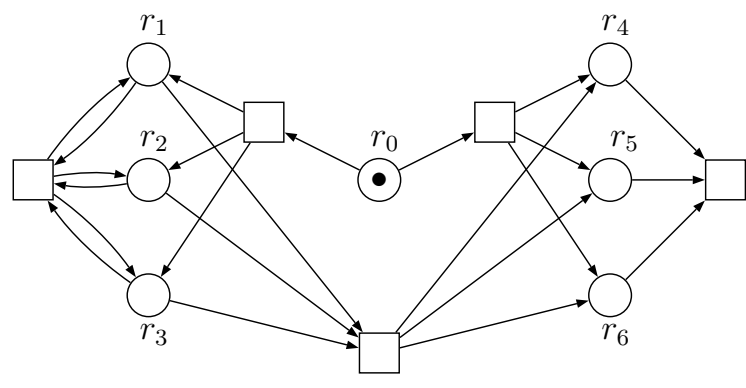

Fig. 1 Petri net $N_{1}$.

We use the notation $(x, y)$-pPN-controlled grammar when $x \in\{f,-\lambda, \lambda\}$ shows the type of a labelling function and $y \in\{r, t\}$ shows the type of a set of final markings. We denote by $p \mathbf{P N}(x, y)$ and $p \mathbf{P N}^{\lambda}(x, y)$ the families of languages generated by $(x, y)$-pPN-controlled grammars without and with erasing rules, respectively, where $x \in\{f,-\lambda, \lambda\}$ and $y \in\{r, t\}$. We also use bracket notation $p \mathbf{P N}^{[\lambda]}(x, y), x \in\{f,-\lambda, \lambda\}, y \in\{r, t\}$ in order to say that a statement holds, in cases with and without erasing rules.

\section{Example 1 Let}

$$
G_{1}=\left(\{S, A, B, C\},\{a, b, c\}, S, R, N_{1}, \beta, M\right)
$$

be a pPN-controlled grammar where $R$ consists of the following productions

$$
\begin{aligned}
& r_{0}: S \rightarrow A B C, \quad r_{1}: A \rightarrow a A, \quad r_{2}: A \rightarrow b B, \\
& r_{3}: A C \rightarrow c C, r_{4}: A \rightarrow a, r_{5}: B \rightarrow b, r_{6}: C \rightarrow c,
\end{aligned}
$$

Fig. 1 illustrates the Petri net $N_{1}$, the place-labelling function $\beta$, and $M$ is defined as $\{(0,0,0,0,0,0,0)\}$. Clearly,

$$
L\left(G_{1}\right)=\left\{a^{n} b^{n} c^{n} \mid n \geqslant 1\right\} \in p \mathbf{P N}(f, t) .
$$

Example 2 Let $G_{2}$ be a pPN-controlled grammar with the rules

$$
\begin{aligned}
& r_{0}: S \rightarrow A B, \quad r_{1}: A \rightarrow a A, \quad r_{2}: B \rightarrow a B, \\
& r_{3}: A \rightarrow b A, r_{4}: B \rightarrow b B, r_{5}: A \rightarrow \lambda, r_{6}: B \rightarrow \lambda
\end{aligned}
$$

Fig. 2 illustrates the Petri net $N_{2}$ and the transitionlabelling function $\beta$ with respect to $G_{2}$. It can be seen that

$$
L\left(G_{2}\right)=\left\{w w \mid w \in\{a, b\}^{*}\right\} \in p \mathbf{P N}(\lambda, t) .
$$

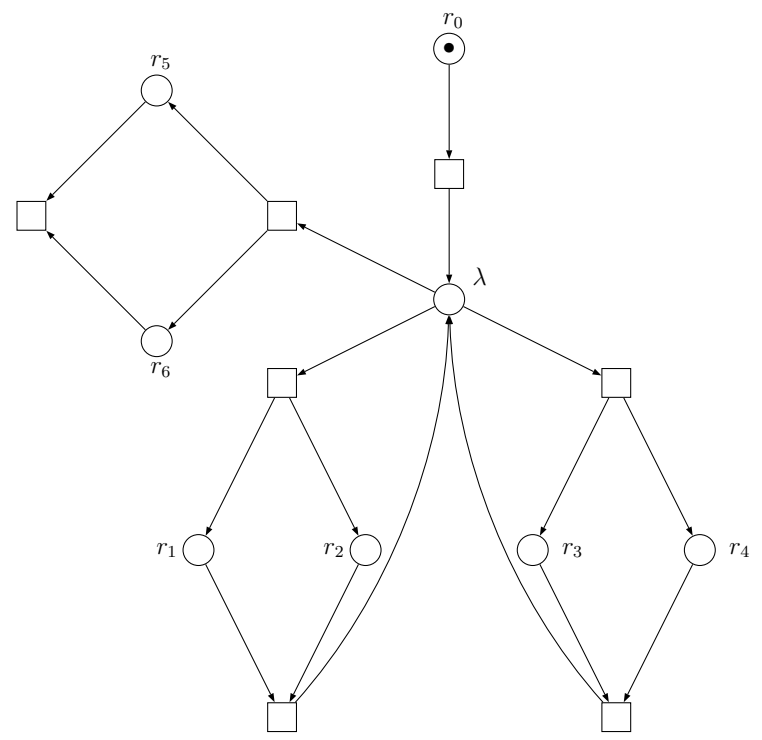

Fig. 2 Petri net $N_{2}$.

\section{LOWER AND UPPER BOUNDS}

The following inclusions immediately follow from the definitions of place-labelled Petri net controlled grammars.

Lemma 1 For $x \in\{f,-\lambda, \lambda\}$ and $y \in\{r, t\}$,

$$
p \mathbf{P N}(x, y) \subseteq p \mathbf{P N}^{\lambda}(x, y) .
$$

Further, we discuss the upper bound for the families of languages generated by pPN-controlled grammars.

Lemma 2 For $y \in\{r, t\}$,

$$
p \mathbf{P N}^{[\lambda]}(-\lambda, y) \subseteq \mathbf{M A T}^{\lambda} \text {. }
$$

Proof: Let $G=(V, \Sigma, S, R, N, \beta, M)$ be an $(-\lambda, y)$ pPN-controlled grammar (with or without erasing rules) and $N=(P, T, F, \phi, \iota)$ where $y \in\{r, t\}$. Let $P=\left\{p_{1}, p_{2}, \ldots, p_{s}\right\}$ and $T_{\varnothing}=\left\{t \in T \mid{ }^{\circ} t=\varnothing\right\}$. Suppose $T-T_{\varnothing}=\left\{t_{1}, t_{2}, \ldots, t_{n}\right\}$. We define the sets of new non-terminals as

$$
\bar{P}=\{\bar{p} \mid p \in P\} \quad \bar{V}=\{\bar{A} \mid A \in V\}
$$

and the homomorphism $h:(V \cup \Sigma)^{*} \rightarrow\left(\bar{V} \cup \Sigma^{*}\right)$ as

$$
h(a)=a, \quad \forall a \in \Sigma h(A)=\bar{A}, \quad \forall A \in V .
$$

Consider $t \in T-T_{\varnothing}$, and let ${ }^{\circ} t=\left\{p_{i_{1}}, p_{i_{2}}, \ldots, p_{i_{k}}\right\}$. We assume that $\beta\left(p_{i_{j}}\right)=A_{i_{j}} \rightarrow \alpha_{i_{j}} \in R, 1 \leqslant j \leqslant k$. Let

$$
h\left(\alpha_{i_{1}} \alpha_{i_{2}} \cdots \alpha_{i_{k}}\right)=x_{1} \bar{B}_{1} x_{2} \bar{B}_{2} \cdots x_{l} \bar{B}_{l} x_{l+1},
$$


where $x_{i} \in \Sigma^{*}, 1 \leqslant i \leqslant l+1$ and $\bar{B}_{j} \in \bar{V}, 1 \leqslant j \leqslant l$.

We associate the following sequences of rules with each transition $t \in T-T_{\varnothing}$,

$$
\begin{array}{r}
\delta_{t, \lambda}: \underbrace{\bar{p}_{i_{1}} \rightarrow \lambda, \ldots, \bar{p}_{i_{1}} \rightarrow \lambda}_{\phi\left(p_{i_{1}}, t\right)}, \ldots, \underbrace{\bar{p}_{i_{k}} \rightarrow \lambda, \ldots, \bar{p}_{i_{k}} \rightarrow \lambda}_{\phi\left(p_{i_{k}}, t\right)} \\
\delta_{t, h}: A_{i_{1}} \rightarrow h\left(\alpha_{i_{1}}\right), A_{i_{2}} \rightarrow h\left(\alpha_{i_{2}}\right), \ldots, A_{i_{k}} \rightarrow h\left(\alpha_{i_{k}}\right) \\
\delta_{t, B}: \bar{B}_{1} \rightarrow B_{1}, \bar{B}_{2} \rightarrow B_{2}, \ldots, \bar{B}_{l} \rightarrow B_{l},
\end{array}
$$

and define the matrix

$$
m_{t}=\left(\delta_{t, \lambda}, \delta_{t, h}, \delta_{t, B}, \delta_{t, X}\right)
$$

with

$$
\delta_{t, X}: X \rightarrow \bar{p}_{1}^{\left|\phi\left(t, p_{1}\right)\right|} \cdot \bar{p}_{2}^{\left|\phi\left(t, p_{2}\right)\right|} \cdots \bar{p}_{s}^{\left|\phi\left(t, p_{s}\right)\right|} \cdot X,
$$

where $X$ is a new non-terminal. We also add the starting matrix

$$
m_{0}=\left(S^{\prime} \rightarrow S \cdot \prod_{p \in P} \bar{p}^{|\iota(p)|} \cdot X\right),
$$

where $S^{\prime}$ is the new start symbol. According to types of the sets of final markings, we consider two cases of erasing rules.

Case $y=r$, for each $p \in P$,

$$
m_{p, \lambda}=(\bar{p} \rightarrow \lambda) m_{X, \lambda}=(X \rightarrow \lambda) .
$$

Case $y=t$, for each $\mu \in M$,

$$
m_{\mu, \lambda}=\left(\mu\left(p_{1}\right), \ldots, \mu\left(p_{s}\right), X \rightarrow \lambda\right),
$$

where $\mu\left(p_{i}\right)=\bar{p}_{i} \rightarrow \lambda, \ldots, \bar{p}_{i} \rightarrow \lambda$ for $i=1,2, \ldots, s$. We consider the matrix grammar $G^{\prime}=\left(V^{\prime}, \Sigma, S^{\prime}, M\right)$, where $V^{\prime}=\left\{S^{\prime}, X\right\} \cup \bar{P} \cup \bar{V} \cup V$ and $M$ consists of all matrices (2) and (3) and matrices (4) for case $y=r$ or matrix (5) for case $y=t$. Let

$$
D: S \stackrel{\pi_{1}}{\Longrightarrow} w_{1} \stackrel{\pi_{2}}{\Longrightarrow} w_{2} \cdots \stackrel{\pi_{d}}{\Rightarrow} w_{d}=w \in \Sigma^{*}
$$

be a derivation in $G$. Then $t_{1} t_{2} \cdots t_{d}$, where $\beta\left({ }^{\circ} t_{i}\right)=\pi_{i}, 1 \leqslant i \leqslant d$, is a successful occurrence sequence in $N$. We construct the derivation $D^{\prime}$ in the grammar $G^{\prime}$ simulating the derivation $D$ as follows; we start the derivation $D^{\prime}$ by applying the matrix (3) and obtain

$$
D^{\prime}: S^{\prime} \stackrel{m_{0}}{\Longrightarrow} S \prod_{p \in P} \bar{p}^{|\iota(p)|} X .
$$

Then, for each transition $t_{i}$ in the successful occurrence sequence $t_{1} t_{2} \cdots t_{d}$, we choose the matrix $m_{t_{i}}$ in $D^{\prime}, 1 \leqslant i \leqslant d$,

$$
\begin{aligned}
D^{\prime}: S^{\prime} \stackrel{m_{0}}{\Longrightarrow} S \prod_{p \in P} \bar{p}^{|\iota(p)|} X \stackrel{m_{t_{1}}}{\Longrightarrow} w_{1} z_{1} \stackrel{m_{t_{2}}}{\Longrightarrow} w_{2} z_{2} X \cdots \\
\quad \cdots \stackrel{m_{t_{d}}}{\Longrightarrow} w_{d} z_{d} X=w z_{d} X,
\end{aligned}
$$

where $z_{i} \in \bar{P}^{*}, 1 \leqslant i \leqslant d$.

The rules $\delta_{t_{i}, h}$ and $\delta_{t_{i}, B}, 1 \leqslant i \leqslant d$, simulate the rules in the multiset $\pi_{i}$, whereas the homomorphism $h$ controls that all rules in $\delta_{t_{i}, h}$ are applied only to $w_{i-1}, 2 \leqslant i \leqslant d$.

By construction, the rules $\delta_{t_{i}, \lambda}$ and $\delta_{t_{i}, X}$ simulate the numbers of tokens consumed and produced in the occurrence of transition $t_{i}$. The number of occurrences of each $\bar{p} \in \bar{P}$ in string $z_{i}$ is the same as the number of tokens in place $p \in P$ after the occurrence of $t_{i}$. Moreover, the number of occurrences of $\bar{p} \in \bar{P}$ in string $z_{d}$ and the number of tokens in place $p \in P$ in a final marking $\mu \in M$ are the same.

Further, to erase $z_{d}$ and $X$, we use the matrices (4) or (5) depending on $y \in\{r, t\}$. Thus $L\left(G^{\prime}\right) \subseteq$ $L(G)$. Using the similar arguments in backward manner, one can show that the inverse inclusion also holds.

With slight modification of the arguments of the proof of the lemma above, we can also show that

Lemma 3 For $y \in\{r, t\}$,

$$
p \mathbf{P N}^{[\lambda]}(\lambda, y) \subseteq \mathbf{M A T}^{\lambda} .
$$

Next, we show that every matrix language can be generated by $(f, t)$ - and $(f, r)$-pPN-controlled grammars.

Lemma 4 For $y \in\{r, t\}$,

$$
\operatorname{MAT}^{[\lambda]} \subseteq p \mathbf{P N}^{[\lambda]}(f, y) .
$$

Proof: Let $G=(V, \Sigma, S, M)$ be a matrix grammar with $M=\left\{m_{1}, m_{2}, \ldots, m_{n}\right\}$, where $m_{i}=$ $\left(r_{i 1}, r_{i 2}, \ldots, r_{i k_{i}}\right), 1 \leqslant i \leqslant n$.

We construct an $(f, t)$-pPN-controlled grammar $G^{\prime}=\left(V \cup\left\{S_{0}\right\}, \Sigma, R \cup\left\{S_{0} \rightarrow S\right\}, S_{0}, N, \beta, M\right)$, where the Petri net $N=(P, T, F, \phi, \iota)$, the place-labelling function $\beta: P \rightarrow R \cup\left\{S_{0} \rightarrow S\right\}$ and the final marking set $M$ are defined as follows.

The sets of places, transitions, and arcs;

$$
\begin{aligned}
P & =\left\{p_{0}\right\} \cup\left\{p_{i j} \mid 1 \leqslant i \leqslant n, 1 \leqslant j \leqslant k_{i}\right\}, \\
T= & \left\{t_{0 i} \mid 1 \leqslant i \leqslant n\right\} \cup\left\{t_{i j} \mid 1 \leqslant i \leqslant n, 1 \leqslant j \leqslant k_{i}\right\}, \\
F= & \left\{\left(p_{0}, t_{0 i}\right),\left(t_{0 i}, p_{i 1}\right),\left(p_{i k_{i}}, t_{i k_{i}}\right),\left(t_{i k_{i}}, p_{0}\right) \mid 1 \leqslant i \leqslant n\right\} \\
& \cup\left\{\left(p_{i j}, t_{i j}\right),\left(t_{i j}, p_{i, j+1}\right) \mid 1 \leqslant i \leqslant n, 1 \leqslant j \leqslant k_{i}-1\right\} .
\end{aligned}
$$


The weight function, $\phi(x, y)=1$ for all $(x, y) \in F$. The initial marking, $\iota\left(p_{0}\right)=1$ and $\iota(p)=0$ for all $p \in$ $P-\left\{p_{0}\right\}$. The transition-labelling function, $\beta\left(p_{0}\right)=$ $S_{0} \rightarrow S$ and $\beta\left(p_{i j}\right)=r_{i j}, 1 \leqslant i \leqslant n, 1 \leqslant j \leqslant k_{i}$. The final marking set, $M=\mathscr{R}(N, \iota)$.

Remark 1 By definition of the Petri net $N$, it is not difficult to see that $\mathscr{R}(N, \iota)$ is a finite set. Thus the cases $y=r$ and $y=t$ coincide.

Let

$$
w_{1} \stackrel{r_{i 1}}{\Longrightarrow} w_{2} \stackrel{r_{i 2}}{\Longrightarrow} \cdots \stackrel{r_{i k_{i}}}{\Longrightarrow} w_{k},
$$

where $m_{i}=\left(r_{i 1}, r_{i 2}, \ldots, r_{i k_{i}}\right) \in M$, be derivation steps of a successful derivation $S \stackrel{*}{\Rightarrow} w \in \Sigma^{*}$ in $G$. Then

$$
w_{1} \stackrel{\left[r_{i 1}\right]}{\Longrightarrow} w_{2} \stackrel{\left[r_{i 2}\right]}{\Longrightarrow} \cdots \stackrel{\left[r_{i k_{i}}\right]}{\Longrightarrow} w_{k}
$$

simulated by (6) and $t_{0 i} t_{i 1} t_{i 2} \cdots t_{i k_{i}}$, is a subsequence of a successful occurrence sequence $v \in$ $\mathscr{R}(N, \iota)$. Thus $L(G) \subseteq L\left(G^{\prime}\right)$. The inclusion $L\left(G^{\prime}\right) \subseteq$ $L(G)$ can also be shown by backtracking the arguments above.

From the lemmas above, the theorem follows.

Theorem 2 For $x \in\{f,-\lambda, \lambda\}$ and $y \in\{r, t\}$,

$$
\text { MAT } \subseteq p \mathbf{P N}(x, y) \subseteq \mathbf{M A T}^{\lambda}, p \mathbf{P N}^{\lambda}(x, y)=\mathbf{M A T}^{\lambda} .
$$

\section{THE EFFECT OF LABELLING STRATEGIES}

In this section, we study the labelling effect to the computational power of pPN-controlled grammars. The following lemma follows immediately from the definition of the languages determined by labelling functions.

Lemma 5 For $y \in\{r, t\}$,

$$
p \mathbf{P N}^{[\lambda]}(f, y) \subseteq p \mathbf{P N}^{[\lambda]}(-\lambda, y) \subseteq p \mathbf{P N}^{[\lambda]}(\lambda, y) .
$$

Further, we prove that the reverse inclusions also hold.

Lemma 6 For $y \in\{r, t\}$,

$$
p \mathbf{P N}^{[\lambda]}(-\lambda, y) \subseteq p \mathbf{P N}^{[\lambda]}(f, y) .
$$

Proof: Let $G=(V, \Sigma, R, S, N, \beta, M)$ be a $(-\lambda, y)$ pPN-controlled grammar (with or without erasing rules), where $N=(P, T, F, \phi, \iota)$.

$$
\text { Let } R=\left\{r_{i}: A_{i} \rightarrow \alpha_{i} \mid 1 \leqslant i \leqslant n\right\},
$$

$P^{+}=\{p \in P \mid(p, t) \in F\}, P^{-}=\{p \in P \mid(p, t) \notin F\}$.
We set the following sets of places, transitions and arcs,

$$
\begin{aligned}
\bar{P}= & \left\{c_{p, t}, c_{p, t}^{\prime} \mid(p, t) \in F\right\}, \\
\bar{T}= & \left\{d_{p, t}, d_{p, t}^{\prime} \mid(p, t) \in F\right\}, \\
\bar{F}= & \left\{\left(p, d_{p, t}\right),\left(d_{p, t}, c_{p, t}\right)\left(c_{p, t}, d_{p, t}^{\prime}\right),\left(d_{p, t}^{\prime}, c_{p, t}^{\prime}\right),\left(c_{p, t}^{\prime}, t\right)\right. \\
& \quad \mid(p, t) \in F\} .
\end{aligned}
$$

We also introduce the new non-terminals and productions for each pair $(p, t) \in F$,

$$
\begin{aligned}
\bar{V}= & \left\{A_{p}, A_{p, t} \mid(p, t) \in F\right\}, \\
\bar{R}=\left\{A \rightarrow A_{p}, A_{p} \rightarrow A_{p, t}, A_{p, t} \rightarrow \alpha\right. & \\
& \left.\quad \mid(p, t) \in F, \beta(p)=A \rightarrow \alpha \in R A_{p, t} \in \bar{V}\right\} .
\end{aligned}
$$

We define the weight function $\bar{\phi}: \bar{F} \rightarrow \mathbb{N}$ as

$$
\begin{aligned}
\bar{\phi}\left(p, d_{p, t}\right) & =\bar{\phi}\left(d_{p, t}, c_{p, t}\right)=\bar{\phi}\left(c_{p, t}, d_{p, t}^{\prime}\right) \\
& =\bar{\phi}\left(d_{p, t}^{\prime}, c_{p, t}^{\prime}\right)=\bar{\phi}\left(c_{p, t}^{\prime}, t\right)=\phi(p, t),
\end{aligned}
$$

where $(p, t) \in F$.

Using the sets and function defined above, we construct an $(f, y)$-place-labelled Petri net controlled grammar $G^{\prime}=\left(V^{\prime}, \Sigma, R^{\prime}, S, N^{\prime}, \beta^{\prime}, M^{\prime}\right)$ with

$V^{\prime}=V \cup \bar{V}$

$R^{\prime}=(R-\{A \rightarrow \alpha \in R \mid \beta(p)=A \rightarrow \alpha,(p, t) \in F\}) \cup \bar{R}$.

The set components of the Petri net $N^{\prime}=$ $\left(P^{\prime}, T^{\prime}, F^{\prime}, \phi^{\prime}, \iota^{\prime}\right)$ are defined as follows.

The sets of places, transitions, and arcs,

$P^{\prime}=P \cup \bar{P}, T^{\prime}=T \cup \bar{T}, F^{\prime}=(F-\{(p, t)\} \in F) \cup \bar{F}$.

The weight function $\phi^{\prime}: F^{\prime} \rightarrow \mathbb{N}$,

$$
\phi^{\prime}(x, y)= \begin{cases}\phi(x, y), & (x, y) \in F-\{(p, t) \in F\}, \\ \bar{\phi}(x, y), & (x, y) \in \bar{F} .\end{cases}
$$

The initial marking $\iota^{\prime}: P^{\prime} \rightarrow \mathbb{N}_{0}$,

$$
\iota^{\prime}(p)= \begin{cases}\iota(p), & p \in P \\ 0, & p \in \bar{P} .\end{cases}
$$

The place-labelling function $\beta^{\prime}: P^{\prime} \rightarrow R^{\prime}$,

$$
\beta^{\prime}(p)= \begin{cases}\beta(p), & p \in P^{-}, \\ A \rightarrow A_{p}, & p \in P^{+},\end{cases}
$$

and for each $c_{p, t}$ and $c_{p, t}^{\prime}$ in $\bar{P}$,

$$
\beta^{\prime}\left(c_{p, t}\right)=A_{p} \rightarrow A_{p, t}, \beta^{\prime}\left(c_{p, t}^{\prime}\right)=A_{p, t} \rightarrow \alpha,
$$


where $\beta(p)=A \rightarrow \alpha \in R$. If $y=r$, then the final marking set $M^{\prime}$ is defined as $M^{\prime}=\mathscr{R}\left(N^{\prime}, \iota^{\prime}\right)$, and if $y=t$, then for every $\mu \in M$, we set $v_{\mu} \in M^{\prime}$, where

$$
v_{\mu}(p)= \begin{cases}\mu(p), & p \in P \\ 0, & p \in \bar{P} .\end{cases}
$$

Let us now consider a successful derivation in $G$,

$$
S \stackrel{E_{1}}{\Longrightarrow} w_{1} \stackrel{E_{2}}{\Longrightarrow} w_{2} \stackrel{E_{3}}{\Longrightarrow} \cdots \stackrel{E_{n}}{\Longrightarrow} w_{n}=w \in \Sigma^{*},
$$

where $E_{i}=\left[r_{i_{1}}, r_{i_{2}}, \ldots, r_{i_{k_{j}}}\right] \subseteq R^{\oplus}, r_{i_{j}}: A_{i_{j}} \rightarrow \alpha_{i_{j}}$, with $\beta\left(p_{i_{j}}\right)=r_{i_{j}}, p_{i_{j}} \in P, 1 \leqslant i \leqslant n, 1 \leqslant j \leqslant k_{i}$. Let

$$
P_{i}^{\prime}=\left\{p_{i_{j}} \mid 1 \leqslant j \leqslant k_{i}\right\} \subseteq{ }^{\circ} t_{i}
$$

for some $t_{i} \in T, 1 \leqslant i \leqslant n\left(t_{i}\right.$ and $t_{j}, 1 \leqslant i \neq j \leqslant n$ are not necessarily distinct). Hence, by definition,

$$
\iota \stackrel{t_{1} t_{2} \cdots t_{n}}{\longrightarrow} \mu, \mu \in M
$$

is the successful occurrence of transitions in $N$. Then, by definition of the set $R^{\prime}$ of the rules, each derivation step $w_{i-1} \stackrel{E_{i}}{\Longrightarrow} w_{i}, 1 \leqslant i \leqslant n$, where $w_{0}=$ $S$, in (7) can be simulated with the following sequence of the derivation steps in the grammar $G^{\prime}$,

$$
\begin{aligned}
& w_{i-1} \stackrel{\left(A \rightarrow A_{i_{1}}\right) \cdot\left(A \rightarrow A_{i_{2}}\right) \cdots\left(A \rightarrow A_{i_{k_{i}}}\right)}{\longrightarrow} w_{i-1}^{\prime} \\
& \stackrel{\left(A_{i_{1}} \rightarrow A_{i_{1}, t_{i}}\right) \cdot\left(A_{i_{2}} \rightarrow A_{i_{2}, t_{i}}\right) \cdots\left(A_{i_{k_{i}}} \rightarrow A_{i_{k_{i}}, t_{i}}\right)}{\longrightarrow} w_{i-1}^{\prime \prime} \\
& \stackrel{\left(A_{i_{1}, t_{i}} \rightarrow \alpha_{i_{1}}\right) \cdot\left(A_{i_{2}, t_{i}} \rightarrow \alpha_{i_{2}}\right) \cdots\left(A_{i_{k_{i}}, t_{i}} \rightarrow \alpha_{i_{k_{i}}}\right)}{\longrightarrow} w_{i} .
\end{aligned}
$$

Correspondingly, by construction of the Petri net $N^{\prime}$, each transition $t_{i}, 1 \leqslant i \leqslant n$, in (8) is extended with the occurrence sequence

$$
d_{i_{1}, t_{i}} d_{i_{2}, t_{i}} \cdots d_{i_{k_{i}}, t_{i}} \cdot d_{i_{1}, t_{i}}^{\prime} d_{i_{2}, t_{i}}^{\prime} \cdots d_{i_{k_{i}}, t_{i}}^{\prime} t_{i},
$$

where

$$
{ }^{\circ} d_{i_{j}, t_{i}}=p_{i_{j}}, d_{i_{j}, t_{i}}^{\circ}={ }^{\circ} d_{i_{j}, t_{i}}^{\prime}=\left\{c_{i_{j}, t_{i}}\right\}, d_{i_{j}, t_{i}}^{\circ}=\left\{c_{i_{j}, t_{i}}^{\prime}\right\} \subseteq t_{i} \text {, }
$$

for all $1 \leqslant i \leqslant n, 1 \leqslant j \leqslant k_{i}$. Thus $L(G) \subseteq L\left(G^{\prime}\right)$.

Consider some successful derivation

$$
S \Rightarrow^{*} w, w \in \Sigma^{*}
$$

in the grammar $G^{\prime}$ with

$$
\iota^{\prime} \stackrel{\cdots+\cdots}{\longrightarrow} \mu, \mu \in M^{\prime},
$$

where $t \in T$. By construction of $N^{\prime}$, in order to enable the transition $t$, the transition $d_{p, t}^{\prime} \in{ }^{\circ} c_{p, t}^{\prime}$, for each $c_{p, t}^{\prime} \in{ }^{\circ} t$ and the transition $d_{p, t} \in{ }^{\circ} c_{p, t}$, for each $c_{p, t} \in{ }^{\circ}\left({ }^{\circ} t\right)$ must be fired. Thus if ${ }^{\circ} t=$ $\left\{c_{p_{1}, t}^{\prime}, c_{p_{2}, t}^{\prime}, \ldots, c_{p_{k}, t}^{\prime}\right\}$, then (12) will contain all the transitions

$$
d_{p_{1}, t}, d_{p_{2}, t}, \ldots, d_{p_{k}, t}, d_{p_{1}, t}^{\prime}, d_{p_{2}, t}^{\prime}, \ldots, d_{p_{k}, t}^{\prime} .
$$

Accordingly, (11) contains the rules

$$
A_{i} \rightarrow A_{p_{i}}, A_{p_{i}} \rightarrow A_{p_{i}, t}, A_{p_{i}, t} \rightarrow \alpha_{i},
$$

where $\beta\left(p_{i}\right)=A_{i} \rightarrow \alpha_{i}, 1 \leqslant i \leqslant k$. Without loss of generality, we can rearrange the order of the occurrence of the transitions in (13) and correspondingly, the order of the application of the rules in (14), and as the result, we construct the occurrence steps and the derivation steps similar to (10) and (9), respectively. Thus the transitions (13) can be replaced with $t$ in the grammar $G$ and the rules (14) can be replaced with the rules $A_{i} \rightarrow \alpha_{i}, 1 \leqslant i \leqslant k$, which results in $L\left(G^{\prime}\right) \subseteq L(G)$.

Lemma 7 For $y \in\{r, t\}$,

$$
p \mathbf{P N}^{[\lambda]}(\lambda, y) \subseteq p \mathbf{P N}^{\lambda}(-\lambda, y) .
$$

Proof: Let $G=(V, \Sigma, R, S, N, \beta, M)$ be a $(\lambda, y)-\mathrm{pPN}$ controlled grammar (with or without erasing rules). Let

$$
P_{\lambda}=\{p \mid \beta(p)=\lambda\}, P_{S}=\{p \mid \beta(p)=S \rightarrow \alpha \in R\} .
$$

We define $(-\lambda, y)$-pPN-controlled grammar

$$
\begin{aligned}
G^{\prime}= & \left(V \cup\left\{S_{0}, X\right\}, \Sigma, S_{0},\right. \\
& \left.R \cup\left\{S_{0} \rightarrow S X, X \rightarrow X, X \rightarrow \lambda\right\}, N^{\prime}, \beta^{\prime}, M^{\prime}\right),
\end{aligned}
$$

where $N^{\prime}=\left(P \cup\left\{p_{0}, p_{\lambda}\right\}, T \cup\left\{t_{0}, t_{\lambda}\right\}, F^{\prime}, \phi^{\prime}, \iota^{\prime}\right)$ with the set of arcs

$$
\begin{aligned}
F^{\prime}=F \cup\left\{\left(p_{0}, t_{0}\right)\right. & \left.,\left(t_{0}, p_{\lambda}\right),\left(p_{\lambda}, t_{\lambda}\right)\right\} \\
\cup & \left\{\left(t_{0}, p\right) \mid \beta(p)=S \rightarrow \alpha \in R\right\},
\end{aligned}
$$

the weight function

$\phi^{\prime}(x, y)= \begin{cases}\phi(x, y), & (x, y) \in F \\ 1, & (x, y) \in\left\{\left(p_{0}, t_{0}\right),\left(t_{0}, p_{\lambda}\right),\left(p_{\lambda}, t_{\lambda}\right)\right\} \\ \iota(p), & (x, y)=\left(t_{0}, p\right), p \in P_{S}\end{cases}$

and the initial marking

$$
\iota^{\prime}(x, y)= \begin{cases}1, & p=p_{0} \\ 0, & p \in P_{S} \\ \iota(p), & p \in P-P_{S}\end{cases}
$$




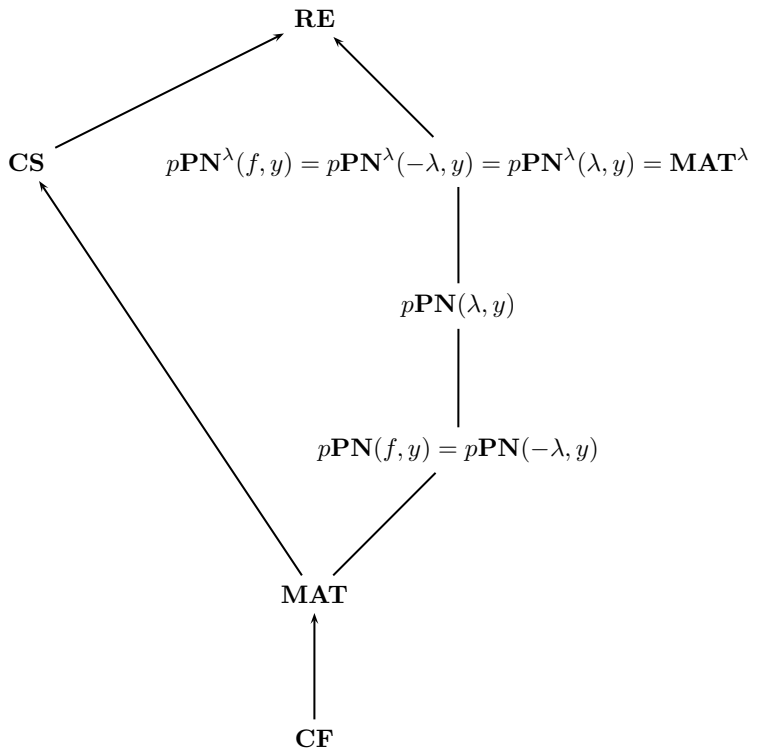

Fig. 3 The hierarchy of the family of languages generated by place-labelled Petri net controlled grammars.

The place-labelling function $\beta$ is modified as

$$
\beta^{\prime}(p)= \begin{cases}\beta(p), & p \notin P_{\lambda}, \\ X \rightarrow X, & p \in P_{\lambda}, \\ X \rightarrow \lambda, & p=p_{\lambda} .\end{cases}
$$

Lastly, when $y=t$, for each final marking $\mu \in M$, we set $v_{\mu} \in M^{\prime}$ as

$$
v_{\mu}(p)= \begin{cases}\mu(p), & P, \\ 0, & p \in\left\{p_{0}, p_{\lambda}\right\} .\end{cases}
$$

Further, it is not difficult to see that $L(G)=$ $L\left(G^{\prime}\right)$.

The following theorem summarizes the results obtained above.

\section{Theorem 3}

$$
\begin{aligned}
p \mathbf{P N}(f, y) & =p \mathbf{P N}(-\lambda, y) \\
& \subseteq p \mathbf{P N}(\lambda, y) \\
& \subseteq p \mathbf{P N}^{\lambda}(f, y) \\
& =p \mathbf{P N}^{\lambda}(-\lambda, y) \\
& =p \mathbf{P N}^{\lambda}(\lambda, y) .
\end{aligned}
$$

By combining the results in Theorems 1, 2 and 3, we obtain the hierarchy of the family of languages generated by place-labelled Petri net controlled grammars.
Theorem 4 The relations in Fig. 3 hold, where the lines (arrows) denote inclusions (proper inclusions) of the lower families into the upper families.

\section{STRUCTURAL PROPERTIES}

In this section, we investigate structural properties of place-labelled Petri net controlled grammars.

\section{A single start place}

Definition 7 Let $G=(V, \Sigma, R, S, N, \beta, M)$ with $N=$ $(P, T, F, \phi, \iota)$ be an $(x, y)$-pPN-controlled grammar, where $x \in\{f,-\lambda, \lambda\}$ and $y \in\{r, t\}$. We say that $N$ has a single start place $p_{0}$ if $\iota\left(p_{0}\right)=1$ and $\iota(p)=0$ for all $p \in P-\left\{p_{0}\right\}$.

Lemma 8 For every $(x, y)$-place-labelled $P N$ controlled grammar $G=(V, \Sigma, R, S, N, \beta, M)$ with a Petri net $N=(P, T, F, \phi, \iota)$, where $x \in\{f,-\lambda, \lambda\}$ and $y \in$ $\{r, t\}$, there exists an equivalent $(x, y)$-pPN-controlled grammar $G^{\prime}=\left(V^{\prime}, \Sigma, R^{\prime}, S^{\prime}, N^{\prime}, \beta^{\prime}, M^{\prime}\right)$ such that the Petri net $N^{\prime}=\left(P^{\prime}, T^{\prime}, F^{\prime}, \phi^{\prime}, \iota^{\prime}\right)$ has a single start place.

Proof: Let $G=(V, \Sigma, S, R, B, \beta, M)$ be a $(x, y)$-pPNcontrolled grammar (with or without erasing rules). We introduce a new place $p_{0}$, a new transition $t_{0}$, and new arcs

$$
\bar{F}=\left\{\left(p_{0}, t_{0}\right)\right\} \cup\left\{\left(t_{0}, p\right) \mid p \in P, \iota(p)>0\right\},
$$

and define the $(x, y)$-pPN-controlled grammar

$$
G^{\prime}=\left(V \cup\left\{S_{0}\right\}, \Sigma, S_{0}, R \cup\left\{S_{0} \rightarrow S\right\}, N^{\prime}, \beta^{\prime}, M^{\prime}\right)
$$

with the Petri net

$$
N^{\prime}=\left(P \cup\left\{p_{0}\right\}, T \cup\left\{t_{0}\right\}, F \cup \bar{F}, \phi^{\prime}, \iota\right),
$$

where the weight function $\phi^{\prime}: F \cup \bar{F} \rightarrow \mathbb{N}$,

$$
\phi^{\prime}(x, y)= \begin{cases}\phi(x, y), & \text { all }(x, y) \in F, \\ \iota(p), & \text { all }(x, y) \in \bar{F},\end{cases}
$$

and the initial marking $\iota^{\prime}: P \cup\left\{p_{0}\right\} \rightarrow\{0,1,2, \ldots\}$,

$$
\iota^{\prime}(p)= \begin{cases}1, & p=p_{0} \\ 0, & p \in P .\end{cases}
$$

Further, the place-labelling function $\beta^{\prime}: P \cup\left\{p_{0}\right\} \rightarrow$ $R \cup\left\{S_{0} \rightarrow S\right\}$ is defined as

$$
\beta^{\prime}(p)= \begin{cases}S_{0} \rightarrow S, & p=p_{0} \\ \beta(p), & p \in P\end{cases}
$$

and for every $\mu \in M$, we set $v_{\mu} \in M^{\prime}$ with $v_{\mu}\left(p_{0}\right)=0$ and $v_{\mu}(p)=\mu(p)$ for all $p \in P$. Then it is not difficult to see that $L(G)=L\left(G^{\prime}\right)$. 


\section{Removal of dead places}

Definition 8 Let $N=(P, T, F, \phi, \iota)$ be a marked Petri net. A place $p \in P$ is said to be dead if $p^{\circ}=\varnothing$.

Lemma 9 For an $(x, y)$-pPN-controlled grammar $G=(V, \Sigma, S, R, N, \beta, M), x \in\{\lambda,-\lambda, f\}$ and $y \in$ $\{r, t\}$, there exists an equivalent $(x, y)-p P N$-controlled grammar $G^{\prime}=\left(V, \Sigma, S, R, N^{\prime}, \beta^{\prime}, M^{\prime}\right)$, where $N^{\prime}$ is without dead places.

Proof: Let $G=(V, \Sigma, R, S, N, \beta, M)$ be an $(x, y)$ place-labelled Petri net controlled grammar with $N=(P, T, F, \phi, \iota)$, where $x \in\{f, \lambda,-\lambda\}$ and $y \in$ $\{r, t\}$. Let

$$
P_{\varnothing}=\left\{p \in P \mid p^{\circ}=\varnothing\right\}, F_{\varnothing}=\left\{(t, p) \in F \mid p^{\circ}=\varnothing\right\} .
$$

We construct an $(x, y)$-pPN-controlled grammar $G^{\prime}=\left(V, \Sigma, S, R, N^{\prime}, \beta^{\prime}, M^{\prime}\right)$, where the Petri net $N^{\prime}$ is obtained from the Petri net $N$ by removing its dead places and the incoming arcs to these places, $N^{\prime}=\left(P-P_{\varnothing}, T, F-F_{\varnothing}, \phi^{\prime}, \iota^{\prime}\right)$, where

$$
\phi^{\prime}(x, y)=\phi(x, y) \text { for all }(x, y) \in F-F_{\varnothing}
$$

and

$$
\iota^{\prime}(p)=\iota(p) \text { for all } p \in P-P_{\varnothing} .
$$

We define the labelling function $\beta^{\prime}:\left(P-P_{\varnothing}\right) \rightarrow R$ by setting

$$
\beta^{\prime}(p)=\beta(p) \text { for all } p \in P-P_{\varnothing} .
$$

For every $\mu \in M$, we set $v_{\mu} \in M^{\prime}$ as

$$
v_{\mu}(p)=\mu(p) \text { for all } p \in P-P_{\varnothing} .
$$

Let

$$
\iota \stackrel{t_{1} t_{2} \cdots t_{n}}{\longrightarrow} \mu, \quad \mu \in M
$$

be a successful occurrence sequence of transitions in $N$. Then, for any place $p \in{ }^{\circ} t_{i}, 1 \leqslant i \leqslant n$, we have $p \notin P_{\varnothing}$. Thus (15) is also successful occurrence sequence in $N^{\prime}$.

\section{A reduction to ordinary nets}

Here, we show that for each pPN-controlled grammar we can construct an equivalent place-labelled ordinary net (pON) controlled grammar.

Lemma 10 Let $G=(V, \Sigma, R, S, N, \beta, M)$ with $N=$ $(P, T, F, \phi, \iota)$ be an $(x, y)-p P N$-controlled grammar, where $x \in\{f,-\lambda, \lambda\}$ and $y \in\{r, t\}$. Then there exists an equivalent $(\lambda, y)$-place-labelled ordinary net controlled grammar $G^{\prime}=\left(V^{\prime}, \Sigma, R^{\prime}, S^{\prime}, N^{\prime}, \beta^{\prime}, M^{\prime}\right)$.
Proof: Let $G=(V, \Sigma, S, R, N, \beta, M)$ with $N=$ $(P, T, F, \phi, \iota)$ be an $(x, y)$-pPN-controlled grammar (with or without erasing rules), where $x \in$ $\{f,-\lambda, \lambda\}$ and $y \in\{r, t\}$. We set

$$
\begin{aligned}
& P^{+}=\bigcup_{(p, t) \in F}\left\{b_{p t}^{i} \mid 1 \leqslant i \leqslant \phi(p, t)\right\}, \\
& P^{-}=\bigcup_{(t, p) \in F}\left\{b_{t p}^{i} \mid 1 \leqslant i \leqslant \phi(t, p)\right\}, \\
& T^{+}=\bigcup_{(p, t) \in F}\left\{d_{p t}^{i} \mid 1 \leqslant i \leqslant \phi(p, t)\right\}, \\
& T^{-}=\bigcup_{(t, p) \in F}\left\{d_{t p}^{i} \mid 1 \leqslant i \leqslant \phi(t, p)\right\},
\end{aligned}
$$

and

$$
\begin{aligned}
& F^{+}=\bigcup_{(p, t) \in F}\left\{\left(p, d_{p t}^{i}\right),\left(d_{p t}^{i}, b_{p t}^{i}\right),\left(b_{p t}^{i}, t\right) \mid 1 \leqslant i \leqslant \phi(p, t)\right\}, \\
& F^{-}=\bigcup_{(t, p) \in F}\left\{\left(t, b_{t p}^{i}\right),\left(b_{t p}^{i}, d_{t p}^{i}\right),\left(d_{t p}^{i}, p\right) \mid 1 \leqslant i \leqslant \phi(t, p)\right\} .
\end{aligned}
$$

We define the $(\lambda, y)$-pPN-controlled grammar $G^{\prime}=\left(V, \Sigma, S, R, N^{\prime}, \beta^{\prime}, M^{\prime}\right)$ with the Petri net $N=$ $\left(P^{\prime}, T^{\prime}, F^{\prime}, \phi^{\prime}, \iota^{\prime}\right)$, where the set of places, transitions, and arcs are constructed as

$P^{\prime}=P \cup P^{+} \cup P^{-}, T^{\prime}=T \cup T^{+} \cup T^{-}, F^{\prime}=F^{+} \cup F^{-}$,

the weight function $\phi^{\prime}: F^{\prime} \rightarrow \mathbb{N}$ is set as $\phi^{\prime}(x, y)=1$ for all $(x, y) \in F^{\prime}$, and the initial marking is defined as

$$
\iota^{\prime}(p)= \begin{cases}\iota(p), & p \in P, \\ 0, & \text { otherwise }\end{cases}
$$

Further, we set the place-labelling function $\beta^{\prime}$ : $P^{\prime} \rightarrow R$ as $\beta^{\prime}\left(b_{p t}^{1}\right)=\beta(p)$ for each $(p, t) \in F$ and $\beta^{\prime}(p)=\lambda$ if $p \in P \cup P^{-} \cup\left(P^{+}-\left\{b_{p t}^{1} \mid(p, t) \in F\right\}\right)$, and define the final markings $v_{\mu} \in M^{\prime}$ when $y=t$ as

$$
v_{\mu}(p)= \begin{cases}\mu(p), & p \in P, \\ 0, & \text { otherwise. }\end{cases}
$$
$L\left(G^{\prime}\right)$.

Further, one can easily show that $L(G)=$

\section{CONCLUSIONS}

In this paper, we defined place-labelled Petri net (pPN) controlled grammars, and investigated their computational power and some structural properties. We showed the followings. pPN-controlled grammars have at least the computational power of matrix grammars without erasing rules and at most the computational power of matrix grammars with erasing rules. The labelling strategies do not effect 
to the generative capacities of pPN-controlled grammars with erasing rules. Although free- and lambdafree-pPN-controlled grammars without erasing rules have the same computational power, the 'lambda' case remains open. The control Petri nets can be reduced to 'canonical forms' without effecting to the generative capacity of pPN-controlled grammars.

The strictness of the inclusions in Theorem 4 is an interesting topic for future research, since it may lead to the solution of a classical open problem, whether $\mathbf{M A T} \subset \mathbf{M A T}^{\lambda}$ or not.

Acknowledgements: The first author would like to thank Universiti Teknologi Malaysia for the UTM Zamalah Scholarship and International Islamic University Malaysia for the financial funding through Endowment B Fund EDW B13-053-0983. The second author is grateful to the Ministry of Education and Research Management Centre, International Islamic University Malaysia for the financial funding through FRGS13-066-0307 and RIGS16-3680532. The third and fourth authors would like to thank the Ministry of Education and Research Management Centre, UTM for the financial funding through Research University Fund Vote No. 08H45.

\section{REFERENCES}

1. Petri CA (1962) Kommunication mit Automaten. $\mathrm{PhD}$ thesis, Univ of Bonn, Germany.

2. Jantzen M (1979) On the hierarchy of Petri net languages. Theor Informat Appl 13, 19-30.

3. Jantzen M (1987) Language theory of Petri nets. In: Brauer W, Reisig W, Rozenberg G (eds) Petri Nets: Central Models and Their Properties, Springer, Berlin, pp 397-412.

4. Hack M (1976) Petri net languages. Computation Structures Group Memo, Project MAC 124, MIT.

5. Ginzburg A, Yoeli M (1980) Vector addition systems and regular languages. J Comput Syst Sci 20, 277-84.

6. Jantzen M, Petersen H (1994) Cancellation in context-free languages: enrichment by reduction. Theor Comput Sci 127, 149-70.

7. Valk R, Vidal-Naquet G (1981) Petri nets and regular languages. J Comput Syst Sci 23, 299-325.

8. Yen HC (1996) On the regularity of Petri net languages. Inform Comput 124, 168-81.

9. ter Beek M, Kleijn J (2002) Petri net control for grammar systems. In: Brauer W, Ehrig H, Karhumäki J, Salomaa A (eds) Formal and Natural Computing, Springer, Berlin, pp 220-43.

10. Farwer B, Kudlek M, Rölke H (2006) Petri-netcontrolled machine models. Tech Rep 274, FBIBericht, Hamburg.

11. Farwer B, Kudlek M, Rölke H (2007) Concurrent Turing machines. Fund Inform 79, 303-17.

12. Farwer B, Jantzen M, Kudlek M, Rölke H, Zetzsche
G (2008) Petri net controlled finite automata. Fund Inform 85, 111-21.

13. Jantzen M, Kudlek M, Zetzsche G (2008) Language classes defined by concurrent finite automata. Fund Inform 85, 267-80.

14. Zetzsche G (2009) Erasing in Petri net languages and matrix grammars. In: Diekert V, Nowotka D (eds) Developments in Language Theory, DLT 2009, Springer, Berlin, pp 490-501.

15. Zetzsche G (2011) A sufficient condition for erasing productions to be avoidable. In: Mauri G, Leporati A (eds) Developments in Language Theory, DLT 2011, Springer, Berlin, pp 452-63.

16. Hauschildt D, Jantzen M (1994) Petri net algorithms in the theory of matrix grammars. Acta Inform 31, 719-28.

17. Marek V, Češka M (2001) Petri nets and randomcontext grammars. In: Proceeding of the 35th Spring Conference Modelling and Simulation of Systems, MOSIS'01, Hradec nad Moravicí, pp 145-52.

18. Dassow J, Turaev S (2008) k-Petri net controlled grammars. In: Martín-Vide C, Otto F, Fernau H (eds) Language and Automata Theory and Applications, LATA 2008, Springer, Berlin, pp 209-20.

19. Dassow J, Turaev S (2008) Arbitrary Petri net controlled grammars. In: Bel-Enguix G, Jiménez-López MD (eds) Linguistics and Formal Languages. Second International Workshop on Non-Classical Formal Languages in Linguistics, Tarragona, Spain, pp 27-39.

20. Dassow J, Turaev S (2009) Grammars controlled by special Petri nets. In: Dediu AH, Ionescu AM, MartínVide C (eds) Language and Automata Theory and Applications, LATA 2009, Springer, Berlin, pp 326-37.

21. Dassow J, Turaev S (2009) Petri net controlled grammars: the power of labeling and final markings. Rom J Inform Sci Tech 12, 191-207.

22. Dassow J, Turaev S (2009) Petri net controlled grammars: the case of special Petri nets. J Univers Comput Sci 15, 2808-35.

23. Dassow J, Turaev S (2010) Petri net controlled grammars with a bounded number of additional places. Acta Cybern 19, 609-34.

24. Stiebe R, Turaev S (2009) Capacity bounded grammars and Petri nets. In: Dassow J, Pighizzini G, Truthe B (eds) 11th International Workshop on Descriptional Complexity of Formal Systems, DCFS 2009, pp 193-203.

25. Stiebe R, Turaev S (2009) Capacity-bounded grammars. J Automata Lang Combinator 15, 175-94.

26. Turaev S (2006) Semi-matrix grammars. In: Second Doctoral Workshop on Mathematical and Engineering Methods in Computer Science, MEMICS 2006, Mikulov, Czechia.

27. Turaev S (2007) Petri net controlled grammars. In: Third Doctoral Workshop on Mathematical and Engineering Methods in Computer Science, MEMICS 2007, Znojmo, Czechia, pp 245-52. 
28. Selamat MH, Turaev S (2010) Grammars controlled by Petri nets with place capacities. In: 2010 Second International Conference on Computer Research and Development, Kuala Lumpur, Malaysia, pp 51-5.

29. Turaev S, Krassovitskiy A, Othman M, Selamat MH (2012) Parsing algorithms for regulated grammars. Int J Math Model Meth Appl Sci 6, 748-56.

30. Turaev S, Krassovitskiy A, Othman M, Selamat MH (2011) Parsing algorithms for grammars with regulated rewriting. In: Recent Researches in Applied Informatics and Remote Sensing: Proceedings of the 11th WSEAS International Conference on Applied Computer Science, pp 103-9.

31. Reisig W, Rozenberg G (1998) Lectures on Petri Nets I: Basic Models, Springer, Berlin.

32. Rozenberg G, Salomaa A (1997) Handbook of Formal Languages, Springer, Berlin.

33. Dassow J, Paun G (1989) Regulated Rewriting in Formal Language Theory, Springer-Verlag, Berlin. 